\title{
Indikation nach medizinischen oder ökonomischen Kriterien?
}

\author{
David Holzmann ${ }^{a}$, Dominik Straumann ${ }^{b}$

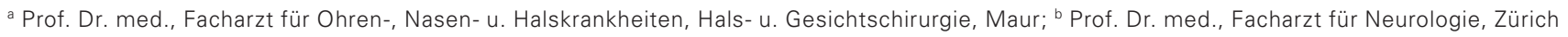

Immer wieder gibt es Sticheleien seitens Politik und Wirtschaft, wonach Ärzte und Spitäler die Patienten endlich nach wirtschaftlichen Kriterien behandeln sollen. Und als Folge dieser Tendenz gibt es bereits viele problematische Entwicklungen in Praxis und Spital, u. a. SwissDRG. Ein bedeutendes Opfer der Ökonomisierung ist die Indikationsstellung. Besonders hier darf man sich nicht von finanziellen, sondern nur von medizinischen Kriterien leiten lassen.

\begin{abstract}
Unter dem Titel «Die Indikation als Kernstück der ärztlichen Identität» wurde im Deutschen Ärzteblatt vom 17.8.2015 ein Interview mit Prof. Dr. med. Giovanni Maio aus Freiburg (Deutschland) veröffentlicht [1]. Kernthema dieses Interviews ist die Auswirkung der zunehmenden Ökonomisierung der Medizin. Maio zeigt in wenigen Sätzen auf, wie sich die ärztliche Indikationsstellung unter dem stetig zunehmenden Druck von Sparmassnahmen und «Optimierungsstrategien» gewandelt hat.

In Studium und Weiterbildung wurde uns gelehrt, dass die Indikation primär nach medizinischen Kriterien zu erfolgen hat. Der Begriff «medizinisch» wurde dabei sehr weit gefasst. So wurden neben den rein medizinisch-wissenschaftlichen auch soziale, psychische und $\mathrm{zu}$ einem geringeren Teil auch wirtschaftliche Faktoren eingeschlossen.
\end{abstract}

Solange wir Ärzte uns immer weiter auseinanderdividieren lassen, wird es in diesem Takt weitergehen.

Die Indikationsstellung ist eine Hauptsäule medizinischen Handelns. Noch expliziter schreibt Maio: «Die medizinische Indikation ist ein Kernstück der ärztlichen Identität. Mit ihr bringt der Arzt zum Ausdruck, dass er dem Patienten nur das empfehlen wird, was für den Patienten im Prinzip zuträglich ist. Zwar wird der Patient selbst entscheiden müssen, aber ein Arzt wird dem Patienten eben nicht etwas empfehlen, was von vornherein nicht gut oder nicht notwendig für den Patienten wäre» [2].

Damit könnte man eigentlich die Diskussion abschliessen, wären da nicht die immer wiederkehrenden Sti- cheleien seitens Politik und Wirtschaft, wonach wir Ärzte und die Spitäler die Patienten endlich nach wirtschaftlichen Kriterien behandeln sollen. Von «Gesundheitsökonomen» - allein schon der Begriff ist ein Widerspruch in sich, denn Gesundheit lässt sich mit keiner Masseinheit und schon gar nicht in Franken messen wurde die ganze Terminologie umgekrempelt. Der Patient wurde zum Kunden, der den Leistungserbringer (oder Leistungsverursacher) mit einer Nachfrage konfrontiert. Dieser wiederum hat dem Kunden seine Leistung nach den WZW-Kriterien (wirksam, zweckmässig, wirtschaftlich) anzubieten. Kann das «gesund» sein?

Diese Frage stellt sich mehr denn je. Denn die Folgen der vorwiegend wirtschaftlichen Steuerung des Gesundheitswesens sind unübersehbar:

- Öffentliche Spitäler, denen die verfassungsmässig zustehenden finanziellen Ressourcen Jahr um Jahr weiter gestrichen werden, versuchen immer mehr, ihr "Angebot» auszubauen.

- Bei immer mehr Privatspitälern wird es offensichtlich, dass der Versicherungsgrad des Patienten Indikationsstellung und Behandlung markant beeinflusst.

Nur schon diese zwei Mechanismen sind volkswirtschaftlich relevant, will man den Zeitungsartikeln glauben, worin Gesundheitspolitiker eine «Mengenausweitung in der medizinischen Versorgung» beklagen. Die Zahl der MR-/Computertomogramme und Operationen (z.B. Hüftgelenkersatz, Sectio u.a.m.) seien in den letzten Jahren exorbitant gestiegen.

Dem versucht die Politik Gegensteuer zu geben, indem sie immer neue Vorschriften erlässt, die einmal mehr das Gegenteil bewirken. Das Beispiel TARMED ist ein 


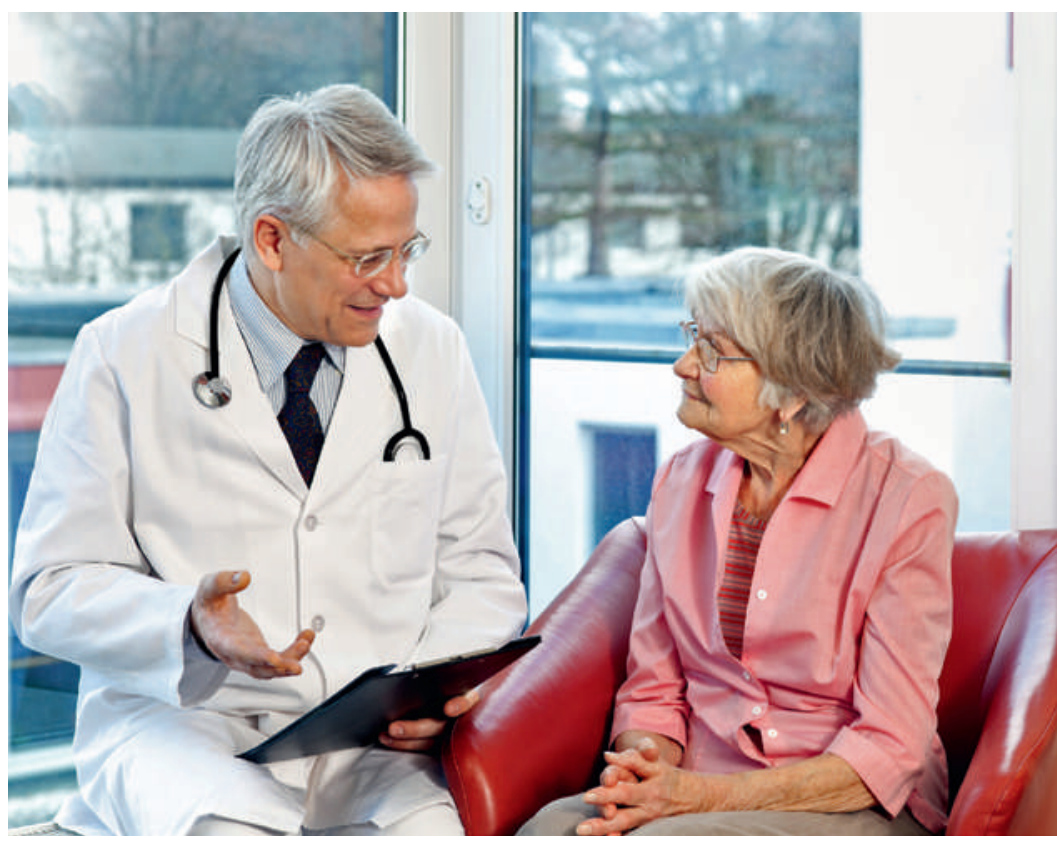

Indikationen sollen wieder mehr nach medizinischen Gesichtspunkten gestellt werden können.
Korrespondenz:

Prof. Dr. med.

David Holzmann

Staubergasse 15

CH-8124 Maur

david.holzmann[at]usz.ch
Paradebeispiel, um dies zu erläutern. Mit dem TARMED köderte die Gesundheitspolitik, damals angeführt von Bundesrätin Dreifuss, die Grundversorger (Hausärzte, Pädiater, allgemeine Internisten, Psychiater) explizit mit der Aussage, im TARMED sollen ärztliche Gespräche mit Patienten höher, dafür die technischen Leistungen tiefer bewertet werden. Die Grundversorger mussten nach gewonnener Abstimmungsschlacht aber schmerzlich zur Kenntnis nehmen, dass sie zu Abstimmungszwecken gründlich missbraucht worden waren. Dafür hatte die Politik und die Verwaltung einen wichtigen Sieg errungen: Hausärzte und Spezialisten sind seither zerstrittener denn je.

Unter dem Vorwand, noch mehr auf die Kostenbremse zu treten, führten später Gesundheitspolitiker Systeme wie die DRG, die im Ausland nachweislich versagt hatten, auch in der Schweiz ein, nachdem sie die «Fallkostenpauschalen" beim Volk mit einer Medienlawine populär gemacht hatten. Kritiker aus den Reihen von uns Ärzten wurden vor die Tür gestellt mit dem Hinweis: «Wir machen nicht die gleichen Fehler wie die Deutschen, sondern wir machen es besser.»

Das Make-up des Grande Euvre, genannt SwissDRG, bröckelt mittlerweile zusehends ab, und das wahre DRG-Gesicht wird erkennbar: Seit die DRG in den Schweizer Spitälern eingeführt wurden, ist ein Run auf "gute Risiken" (junge Patienten, Patienten ohne Komorbiditäten etc.) ausgebrochen. Öffentliche Spitäler «tummeln» sich inzwischen wie die Privatspitäler auch im Gesundheitsmarkt und preisen ihre Angebote mit Hochglanz-Broschüren und auf attraktiven, aber inhaltsleeren Webseiten an.

Ökonomische Kriterien bestimmen also auch in der Schweiz immer mehr das ärztliche Handeln. Wie Giovanni Maio richtig feststellte, ist ein bedeutendes Opfer die Indikationsstellung. Es ist eine menschliche Grundkonstante, dass immer dann, wenn viel Geld im Spiel ist, die Verlockung gross ist, für sich selbst noch ein grösseres Stück vom Kuchen zu ergattern. Dieser Verlockung zu widerstehen, erfordert ein gesundes Mass an Charakterfestigkeit und die ethische Einsicht, dass wir uns in erster Linie für das Wohl aller Menschen, d.h. für das Gemeinwohl, einsetzen sollten. Übersetzt auf die Gesundheitsversorgung hiesse das: Der Arzt lässt sich in der Indikationsstellung nicht von finanziellen, sondern von medizinischen Kriterien leiten. Der Begriff «medizinisch» kann, wie oben erwähnt, durchaus weit gefasst werden. Ärztliches Handeln muss aber auf die Volksgesundheit, das Gemeinwohl, ausgerichtet sein. Finanzieller Profit bzw. Gewinn kann nicht die primäre Zielsetzung sein.

Die FMH - unsere Standesorganisation - schreibt in ihrem Porträt: «Alle Patientinnen und Patienten in der Schweiz sollen einen gerechten Zugang zu einer qualitativ hochstehenden und finanziell tragbaren medizinischen Versorgung haben - dafür setzt sich die FMH ein.» Heute sind wir weiter von diesem Ziel entfernt als noch vor 30 Jahren. Ein Hauptgrund dafür liegt in der zunehmenden Fremdbestimmung von uns Ärzten durch Reformen, die uns von Politik, Wirtschaft und Verwaltung aufoktroyiert werden. Die Verführung und Korrumpierbarkeit durch finanzielle «Aufbesserungen" darf in diesem inzwischen kranken System nicht unterschätzt werden. Solange wir Ärzte uns immer weiter auseinanderdividieren lassen, wird es in diesem Takt weitergehen. Ein Zusammenhalt der Ärzteschaft ist die unabdingbare Voraussetzung, Indikationen wieder mehr nach medizinischen Gesichtspunkten stellen zu können und den "ökonomistischen Turnübungen» seitens Politik und Wirtschaft zu widerstehen. Vergessen wir nicht: Noch ist der Patient unser treuester Verbündeter. Dieses Vertrauen dürfen wir nicht missbrauchen.

\footnotetext{
Literatur

1 Deutsches Ärzteblatt vom 17.8.2015

2 Prof. Dr. med. Giovanni Maio: Geschäftsmodell Gesundheit-Wie der Markt die Heilkunst abschaff -Suhrkamp Taschenbuch 4514 Suhrkamp Verlag Berlin 2014
}

Bildnachweis

Belahoche | Dreamstime.com 